\title{
Lithographic manufacturing of adaptive optics components
}

R. Phillip Scott, Madison Jean, Lee Johnson, Ridley Gatlin, Ryan Bronson, et al.

R. Phillip Scott, Madison Jean, Lee Johnson, Ridley Gatlin, Ryan Bronson, Tom Milster, Michael Hart, "Lithographic manufacturing of adaptive optics components," Proc. SPIE 10401, Astronomical Optics: Design, Manufacture, and Test of Space and Ground Systems, 104011C (5 September 2017); doi: $10.1117 / 12.2276508$

SPIE Event: SPIE Optical Engineering + Applications, 2017, San Diego, California, United States 


\title{
Lithographic manufacturing of adaptive optics components
}

\author{
R. Phillip Scott, Madison Jean, Lee Johnson, Ridley Gatlin, Ryan Bronson, \\ Tom Milster, Michael Hart \\ College of Optical Sciences, University of Arizona, 1630 E University Blvd, Tucson, AZ, 85721, USA
}

\begin{abstract}
Adaptive optics systems and their laboratory test environments call for a number of unusual optical components. Examples include lenslet arrays, pyramids, and Kolmogorov phase screens. Because of their specialized application, the availability of these parts is generally limited, with high cost and long lead time, which can also significantly drive optical system design. These concerns can be alleviated by a fast and inexpensive method of optical fabrication. To that end, we are exploring direct-write lithographic techniques to manufacture three different custom elements. We report results from a number of prototype devices including 1,2, and 3 wave Multiple Order Diffractive (MOD) lenslet arrays with $0.75 \mathrm{~mm}$ pitch and phase screens with near Kolmogorov structure functions with a Fried length $r_{0}$ around $1 \mathrm{~mm}$. We also discuss plans to expand our research to include a diffractive pyramid that is smaller, lighter, and more easily manufactured than glass versions presently used in pyramid wavefront sensors. We describe how these components can be produced within the limited dynamic range of the lithographic process, and with a rapid prototyping and manufacturing cycle. We discuss exploratory manufacturing methods, including replication, and potential observing techniques enabled by the ready availability of custom components.
\end{abstract}

Keywords: adaptive optics, wavefront sensor, manufacturing, turbulence

\section{INTRODUCTION}

While the field of adaptive optics is not new, there is still much progress to be made. Because the field is relatively small and mostly limited to astronomical purposes, components for the field are manufactured typically only in small quantities and are expensive. While some vendors use classic lithography techniques ${ }^{1}$, thin film deposition ${ }^{2}$, or machine milling to get the pattern needed, we are exploring the use of the Maskless Lithography Tool (MLT) developed at the University of Arizona to quickly and simply develop a range of products, both old and new, for the field. Thus far our research has focused on generating phase screens representative of Kolmogorov turbulence and generating lenslet arrays. Because of the limited dynamic range of the MLT, a particular focus of the investigation is the practicality of Multi-Order Diffractive (MOD) structures to create lenslet arrays with higher power than the dynamic range would otherwise allow. We are also considering the potential of the MLT to create diffractive pyramids for wavefront sensing in narrow wavelength bands.

The main motivation for this research is to enable rapid prototyping as well as cost effective production of AO components. With the MLT multiple parts can be manufactured from a design within a day, and the equipment requirements are relatively modest.

\subsection{Lithographic process}

The MLT at the University of Arizona College of Optical Sciences is a direct-write, continuous profile laser lithography tool capable of exposing high resolution grayscale patterns over a $25 \mathrm{~mm}$ square in as little as 60 seconds. The core of the MLT exposure system consists of a Sabre Argon Ion laser (Coherent, Santa Clara, CA) emitting up to $1.3 \mathrm{~W}$ at $363.8 \mathrm{~nm}$, an 8-bit acousto-optic modulator (AOM), and a 12-sided polygon mirror on an air bearing spindle. This creates a flying spot exposure configuration, which, in conjunction with motorized sample stages, allows for very fast grayscale exposure.

Before modulation, the UV beam is aligned using a closed loop servo system consisting of two quad-cell detectors and two piezo controlled steering mirrors. The beam is then modulated by the AOM and directed to the polygon mirror rotating at $3000 \mathrm{rpm}$ where it is converted to a scanline. Scanline optics are employed to flatten the scanline prior to passing through a $3 \mathrm{X}$ afocal reduction relay which produces an approximately $25 \mathrm{~mm}$ long scanline with a $2.1 \mu \mathrm{m}$ spot at the sample plane. The sample stages consist of precision motorized Aerotech $\mathrm{x}$ and $\mathrm{y}$ stages for positioning as well as a Standa $\mathrm{Z}$ stage for focus adjustment. Focusing feedback is achieved by retroreflection from the sample plane. As the beam scans in the $\mathrm{x}$ direction, the y stage moves continuously such that the next scanline will be $2.1 \mu \mathrm{m}$ offset, continuing until the full sample is exposed.

Astronomical Optics: Design, Manufacture, and Test of Space and Ground Systems, edited by Tony B. Hull, Dae Wook Kim, Pascal Hallibert, Gary B. Hughes, Ronald G. Pirich, Proc. of SPIE Vol. 10401, 104011C - @ 2017 SPIE - CCC code: 0277-786X/17/\$18 - doi: 10.1117/12.2276508 
Operation of the system is controlled by a custom Labview software interface which facilitates control of the stages, the polygon mirror, and the AOM as well as data output. Synchronization between data output to the AOM, the rotation of the polygon, and the position of the y-stage is calibrated and constantly checked by an optical signal picked off from the start of each scan line. Data is input to the software in the form of grayscale bitmap files of up to 12,000 by 12,000 pixels with 8-bit depth. The depth of the focus of the exposure beam is approximately $\pm 40 \mu \mathrm{m}$. Using multiple exposure patterns and complimentary focus adjustment, the MLT is capable of exposing patterns on non-planar surfaces such as spheres or aspheres as well.

Our fabrication process begins by exposing the desired pattern into the photoresist (Fujifilm AP2210B Polyimide) laid down on a BK7 substrate. Using this material, the MLT etches $17.5 \mathrm{~nm}$ per step leading to a maximum depth of $4.48 \mu \mathrm{m}$ per pass. After etching, the part can be used directly, but eventually the photoresist can be expected to fail, flaking off in chips like dried paint. The beginnings of this process can be seen in Figure 1 which shows the edge of one of the lenslet arrays under a white light interferometer. A more robust method uses the etched part to create a silicone mold, a negative copy of the piece, from which multiple positive copies can be replicated in epoxy. These final parts have potentially unlimited lifetime, and duplicates can be produced rapidly and very inexpensively.

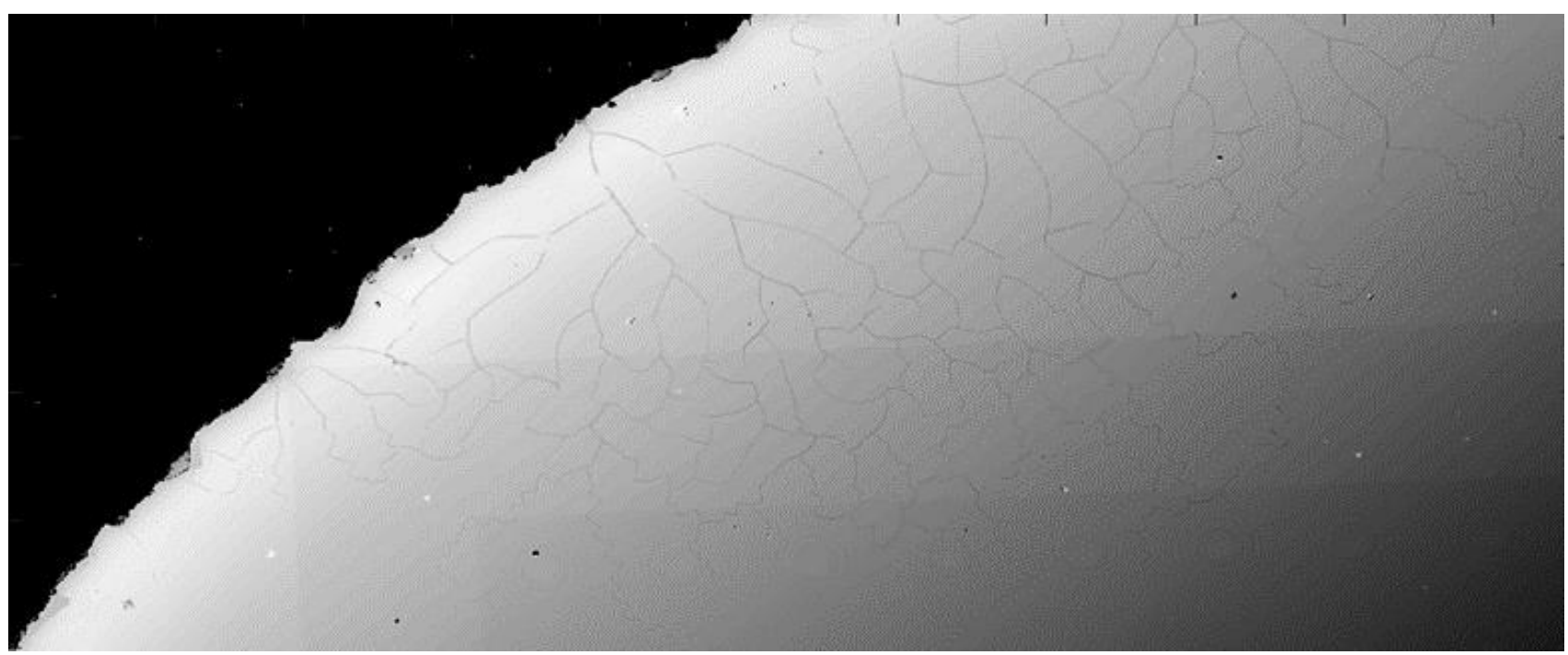

Figure 1: Cracking of the photoresist near the edge of a $25 \mathrm{~mm}$ round plate.

\section{MANUFACTURING AND ANALYSIS OF AO COMPONENTS}

Using the MLT described in Section 1.1, we have made a variety of parts applicable to laboratory demonstrations of AO. We began by investigating Kolmogorov phase screens to reproduce turbulence in a laboratory environment. An initial 21 $\mathrm{mm}$ square turbulence piece was followed by larger screens made by stitching multiple square patches of turbulence together. We also investigated new designs of classic optical components: Shack Hartmann lenslet arrays and pyramids for wavefront sensing. The design and effectiveness of these pieces are discussed below.

\subsection{Kolmogorov phase plates}

We have initially focused on the development of Kolmogorov phase screens with the intent to produce parts up to $75 \mathrm{~mm}$ in size. We developed software to generate phase aberrations obeying Kolmogorov statistics. Overall tilt is subtracted from the phase screen since it is unnecessary to our optical tests and reduces the dynamic range available for writing the higher order aberration. Nevertheless, given that the phase screen dimensions are expected to be approximately $100 r_{0}$, the 8-bit depth of the MLT is insufficient to afford adequate resolution of the optical path length. To compress the dynamic range of the screen within the MLT's capability, we apply a high-pass filter, removing the lowest spatial frequencies which have the largest amplitude. Since the beam footprints we intend to use will be only 10-20r $r_{0}$ in diameter, the filtering has little effect on the statistical distribution of the instantaneous phase disturbance within the beam. 
An example of a 10,000 $\times 10,000$ pixel phase screen generated in this way is shown in Figure 2. Using the MLT we etched two such patterns into photoresist to create $21 \mathrm{~mm}$ square screens with 8 bit depth, a $2.1 \mu \mathrm{m}$ lateral step size, and $r_{0}$ values of $1.5 \mathrm{~mm}$ and $2 \mathrm{~mm}$. This is almost as large a piece as the MLT can fabricate in a single pass. Larger pieces can be made by stitching together sections of a pattern, writing one section at time, and physically moving the substrate in the MLT between sections. To test this process, a second screen was generated from a pattern that was 20,000 pixels square and split into four $10 \times 10 \mathrm{kpx}$ screens. A white light interferometer was used to examine the seam between the sections. Figure 3 shows an example where part of the screen was etched twice, creating a crevice in the phase plate. This effect can be avoided through more careful alignment of the MLT and the phase screen as the part is adjusted between passes.

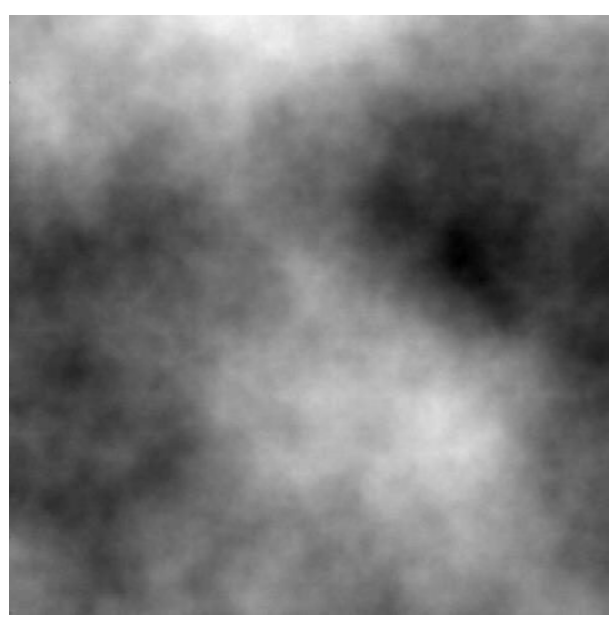

Figure 2: Example of a Kolmogorov turbulence distribution generated via MATLAB.

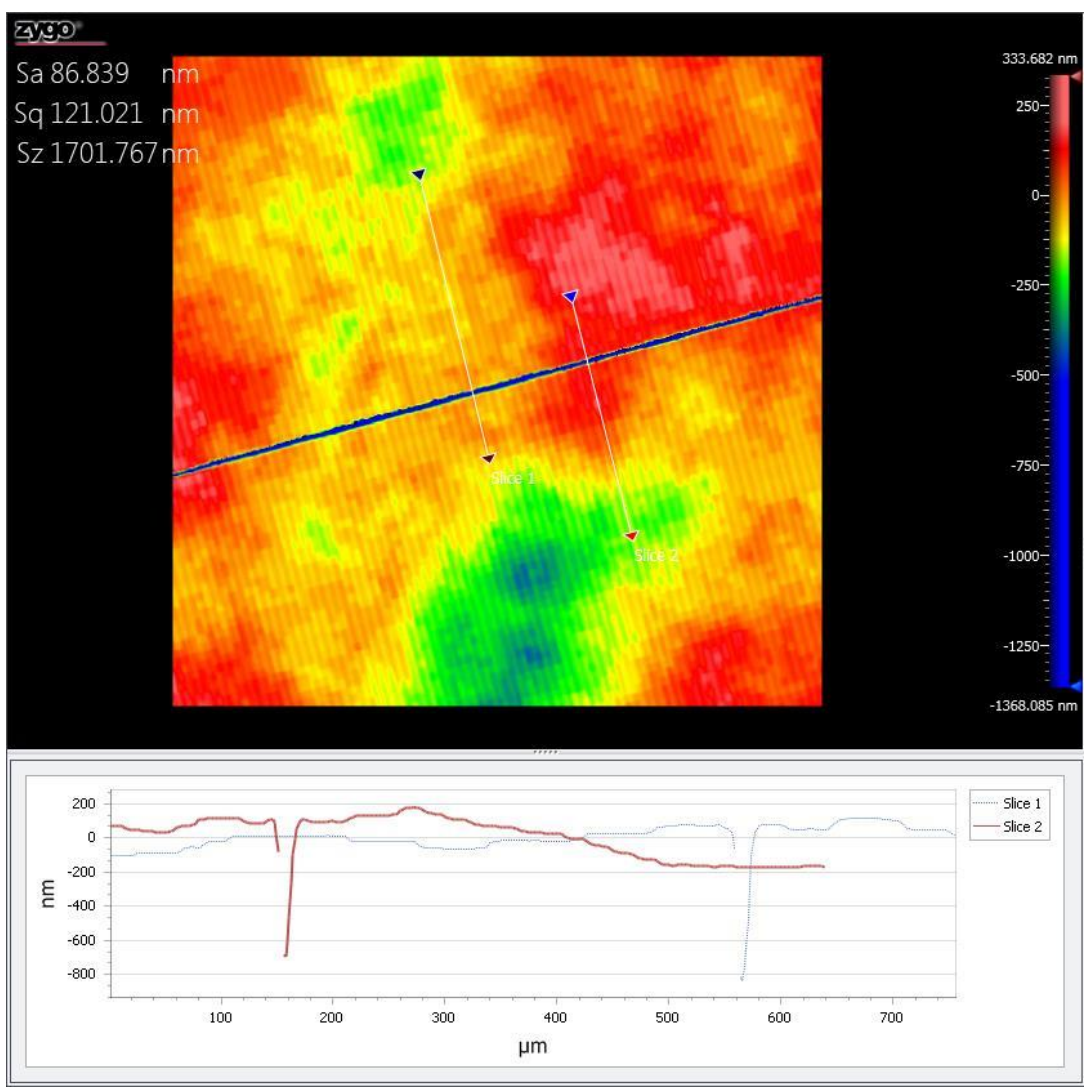

Figure 3: White light interferometer measurement across a seam in stitched phase plate screens. 
The quality of the screens was assessed by measuring the structure function of the phase imparted by them. Phase shifting interferometry yielded the wavefront in transmission. From the wavefront phase map $\emptyset(x)$, we calculated a structure function using Equation 1.

$$
D_{\varnothing}(\Delta x)=\sum(\varnothing(x)-\emptyset(x-\Delta x))^{2}
$$

The structure function of aberration caused by Kolmogorov turbulence is expected to follow Equation 2.

$$
D_{\varnothing}(r)=6.88\left(\frac{r}{r_{0}}\right)^{5 / 3}
$$

Once the 2D structure function of our phase plates were calculated, the result was radially averaged and compared to the form in Equation 3. This is done by linearizing the equation through a logarithm as shown in Equation 3, and fitting the result with a line.

$$
\ln \left(D_{\varnothing}\right)=\frac{5}{3} \ln (r)+\ln \left(\frac{6.88}{r_{0}^{5 / 3}}\right)=a \ln (r)+b
$$

If the slope of the line $a=5 / 3$, then the phase aberration is indeed representative of physical turbulence ${ }^{3}$. The value of $r_{0}$ may also be inferred from the fit via Equation 4.

$$
r_{0}=\left(6.88 * e^{-b}\right)^{1 / a}
$$

Using this method, the two original phase screens were fit with slopes of $a=1.60$ and $a=1.65$ demonstrating nearKolmogorov behavior at scales below $1.5 \mathrm{~mm}$. Using the fit parameters, it was determined that the plates had $r_{0}$ values of 1.5 and $2 \mathrm{~mm}$ respectively. We had been unable to predict this value in advance because of uncertainties in the MLT process. It was also determined that the radially averaged structure function of our stitched screen, shown in Figure 4, had an average slope of 1.621 on scales less than $2 \mathrm{~mm}$, very close to the expected value. The average value of $r_{0}$ was $0.79 \mathrm{~mm}$. Because of changes in the process between the original screens and the stitched screen, we were once more unable to

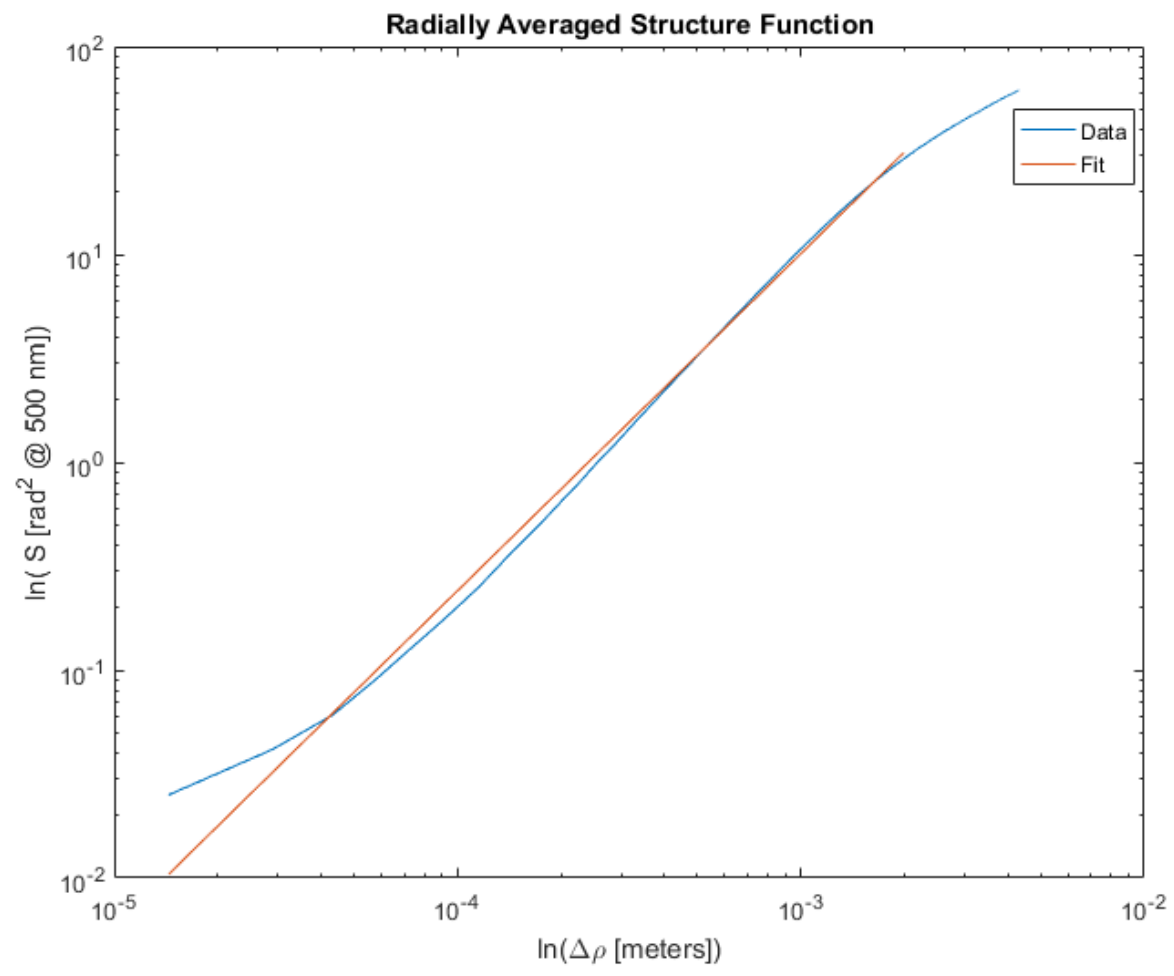

Figure 4: Radially averaged structure function printed on a logarithmic scale. The linear fit has parameters $\mathrm{a}=1.621$ and $\mathrm{b}=13.51$ yielding an $r 0$ value of $0.79 \mathrm{~mm}$ 
predict this value, but are using this measurement as a calibration around which to design future phase screens. The average slope of 1.62 demonstrates that the phase screens do exhibit near-Kolmogorov properties on small scales and confirms that a usable value of $r_{0}$ can be achieved using the MLT. Given this result, we are satisfied that the phase plates that we can and have produced will suffice to simulate realistic turbulence in a laboratory setting.

\subsection{MOD lenslet arrays}

Lenslet arrays are a crucial component of the majority of AO systems, which rely on Shack-Hartman wavefront sensors. While the industry standard is refractive lenslet arrays, we have been investigating Multi-Order Diffractive (MOD) lenslets as a means of rapid fabrication while accommodating the limited dynamic range of the MLT. To test the practicality and capabilities of these lenslets we created three arrays at roughly f/20 with OPD steps of 1,2, and 3 waves. A single lenslet of pitch $0.75 \mathrm{~mm}$ from each array was imaged using a white light interferometer and is shown in Figure 5. Each array comprised a $15 \times 15$ repeating pattern of these lenslets.

Our analysis of the lenslet arrays ${ }^{4}$ began by illuminating the array with collimated light from a spatial filter. We recorded the resulting point spread functions (PSFs) from each lenslet on a FLIR Flea3 camera. Equation 5 shows the expected Fraunhoffer diffraction pattern from a square aperture that would be manifest at the focus of a lens.

$$
P S F \propto \operatorname{sinc}^{2}\left(\frac{x}{\lambda(f / \#)_{x}}\right) * \operatorname{sinc}^{2}\left(\frac{y}{\lambda(f / \#)_{y}}\right)
$$

Knowing that each lenslet was inside of a square aperture, we then fit the resulting PSFs with the expected diffraction pattern to measure the $\mathrm{f} / \#$ of each individual lenslet and to track the variation between lenslets. The resulting analysis showed good agreement across lenslets in a single array giving confidence in the manufacturing process. The one wave lenslet had an average f/\# of 21.9 across 88 of its lenses with a standard deviation of 0.86 . The two wave lenslet had an average f/\# of 21.7 with a standard deviation of 1.3. Finally, the three wave lenses exhibited an average f/\# of 19.7 with a standard deviation of 0.56 . These results indicate that for each given array, the lenslets were fairly homogeneous in $\mathrm{f} / \#$.
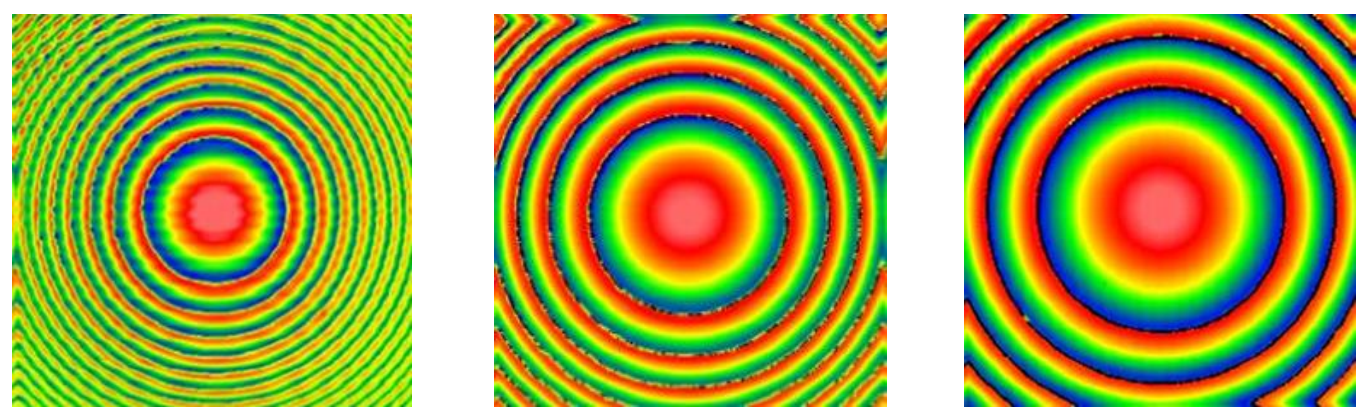

Figure 5: White light interferometer images of MOD lenslets with OPD steps of 1 (left), 2 (center), and 3 (right) waves.

\subsubsection{Chromatic testing}

While the results from the analysis above are promising and give us confidence in the production quality of MOD lenslets and their utility in a narrow band regime, we realize that performance from such a device will suffer with broadband sources. The diffractive nature of the optic means that one would expect the focal length to shift as a function of wavelength more strongly than traditional refractive optics, and that there also would be multiple focal positions for each wavelength. This also means that at a given wavelength not all of the energy will be found at a single focus resulting in less focused light on the detector at a given focal plane. While the strong chromatic aberration is a concern in putting these lenslet arrays to practical use, it will not be a problem in AO systems operating with laser beacons. Even AO systems that rely on broad-band sources will not be adversely affected provided that the blur caused by longitudinal chromatic aberration is smaller than the seeing limit.

To fully understand the effect of different wavelengths on the MOD lenslets, we devised an experiment to measure the focal shift as a function of wavelength. By collimating the output of a monochromator that is illuminated with a supercontinuum laser, we were able to illuminate the lenslets with an adjustable narrow band of collimated light. Using a 
motorized translation stage with a FLIR Grasshopper camera, we captured images of the PSFs created by the lenslet arrays as a function of focal position for each wavelength. The data from this experiment are shown in Figure 6 for the three lenslet arrays that we've manufactured. Also in Figure 6, represented by solid lines, are the theoretical curves for the chromatic focal shift of a MOD lens. The lenses were designed to operate at $638 \mathrm{~nm}$ and at points outside of the design wavelength, we can typically measure at least two focal positions for any given wavelength. It should be noted that the diffraction efficiency is not the same at each focus for a given wavelength and therefore the power is not divided evenly. One of the focal positions will be brighter than the others.

Our measurements confirmed that multiple diffraction orders for a given wavelength appear as expected. Figure 6 shows that the 1 and 2-wave lenslets match the theory very well, but the 3 -wave lenslet departs from the theoretical prediction at long focal positions and high wavelengths according to our measurements. It is also observed that while the data in the 3wave lenslet follows the trend of the theoretical curves, focal positions appear later and extend further than expected with regard to wavelength. We believe this is due to a variation in the manufacturing of this array, a theory that is supported by the relatively large difference in $\mathrm{f} / \#$. We are investigating this possibility, but as of this writing have been unable to determine the underlying cause. While the data affirm the effects of chromatic aberration and blur due to multiple focal modes, we found that over small wavelength bands $(25-50 \mathrm{~nm})$ the effects from multiple focal orders can be negligible provided operation is near the design wavelength. In short, given a specific design wavelength, these lenslets perform admirably for narrow band applications, but suffer much more aberration in general than refractive lenslets as broader band sources are used.

\section{FUTURE WORK}

\subsection{Replication}

We have begun to explore the replication process mentioned in Section 1.1 using a silicone mold to fabricate Kolmogorov phase screens in epoxy. We are now working with the epoxy replicas to ensure that the original pieces are replicated completely and accurately. While we have started this process, it is still in its infancy and needs continued work. Given preliminary results, we believe that replication of phase screens and other AO components will prove quite possible. There is some concern with the ability to replicate the small, sharp features of diffractive components with enough detail. That will be an important next step in our investigation.

\subsection{Diffractive pyramids}

The investigation of diffractive lenslets led us to consider other diffractive AO parts. One such that is particularly difficult to produce is the key component in the pyramid wavefront sensor. This piece is traditionally made either as a monolithic glass piece or by positioning two roof prisms together to very tight tolerances. Using the MLT and our established processes, we aim to create a diffractive solution to this problem that will create a smaller, lighter pyramid etched onto a single piece of glass. Because the MLT uses step sizes of $2.1 \mu \mathrm{m}$, the sharpness of the pyramid edges can be increased by nearly a factor of five compared to glass without the risk of chipping, leading to a decrease in light diffracted from the seam in the pyramid faces. The sharp edges will also ease the design of the optics around the pyramid since faster focal ratios can be used without unacceptable loss of light in the image placed on the pyramid tip. The diffraction efficiency from the stepped sides is theoretically greater than $99.99 \%$ alleviating another concern with this approach ${ }^{5}$.

Another advantage is that the diffractive pyramid will be formed from a single piece of material reducing potential stresses from gluing and CTE mismatch as well as eliminating the alignment tolerances that bonding prisms presents. Compared to conventional designs, these pieces will offer improvements in accuracy, size, and weight. While the design for the diffractive pyramid is complete, manufacturing has not yet begun. Figure 7 shows the design that will be implemented with the MLT. 

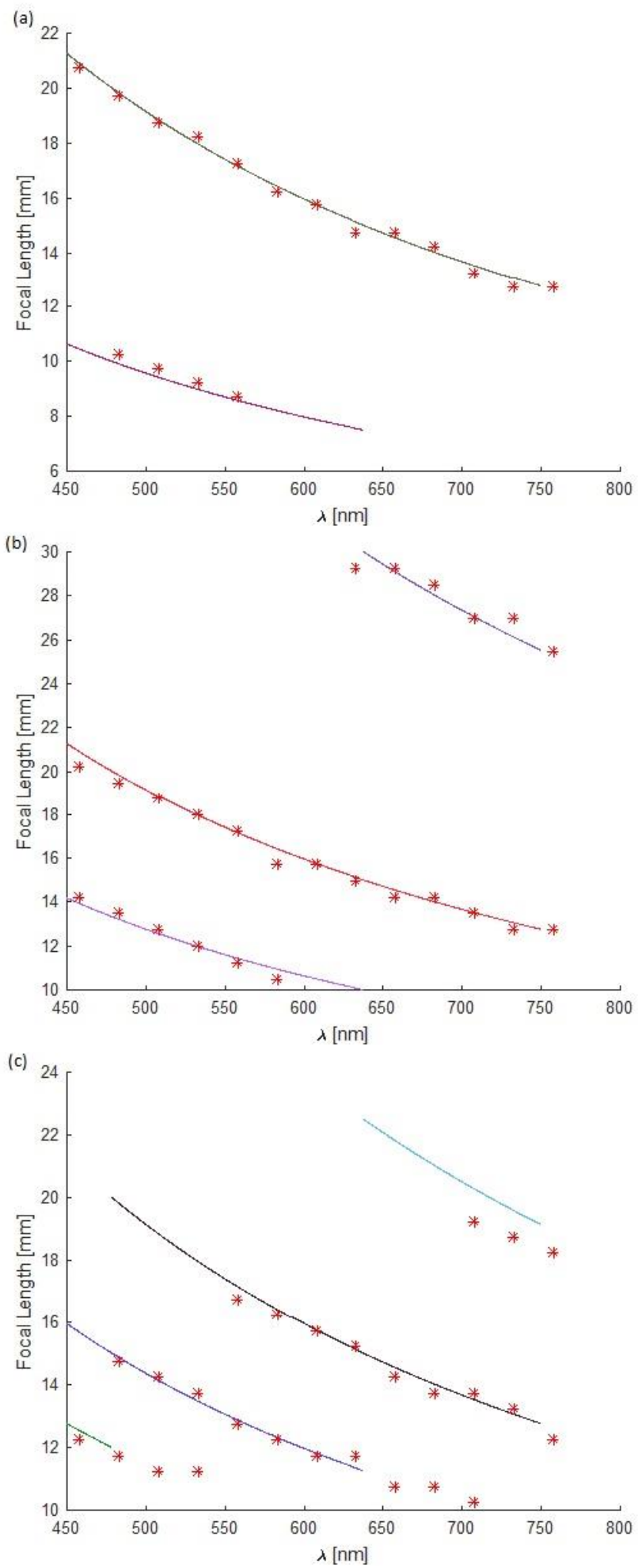

Figure 6: Theoretical (solid lines) and experimental data (points) for the three manufactured lenslet arrays: (a) step sizes of 1 wave of OPD, (b) step sizes of 2 waves of OPD, and (c) step sizes of 3 waves of OPD. 

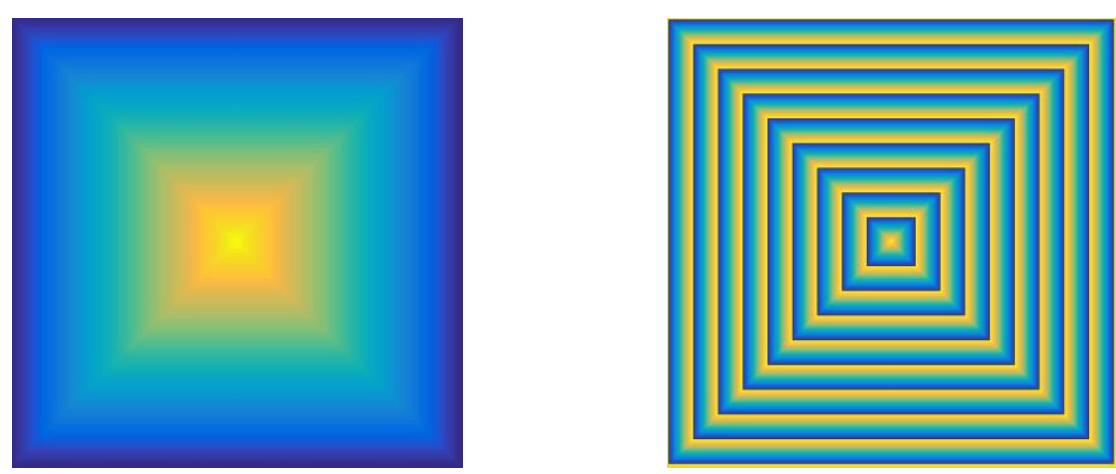

Figure 7: (Left) Phase map of a typical pyramid used for wavefront sensing. (Right) Phase map of a diffractive pyramid with the same deviation angle.

Once fabrication of pyramids with the MLT is demonstrated, the technique can be extended to place multiple pyramids on a single plate. Much in the manner of the slit mask for a multi-object spectrograph, a plate could be rapidly made to place several pyramids at arbitrary locations to match multiple guide stars in a single scene. In this way, the layer-oriented approach to multi-conjugate $\mathrm{AO}$ could be implemented with significant cost savings in the system hardware. ${ }^{6}$ Due to the low cost, low profile, and fast turn time of this technique, it will be possible to design a custom plate prepared with multiple pyramids for a unique patch of sky allowing multiple guide stars each to be analyzed by their own pyramid. This is challenging given the size and cost of current technology and would provide a boost to SNR to single-conjugate AO systems as well as the possibility of using pyramids for tomographic AO techniques.

\subsection{Improving dynamic range}

The dynamic range of the MLT imposes a limitation on the fabrication of optical components. To overcome the limitation we have to date relied on the MOD approach to make the lenslet arrays and high pass filtering for the Kolmogorov phase screens. Another method that would avoid such compromises altogether, but has yet to be tested, would be the approach used by High Dynamic Range (HDR) equipment. A sequence of masks is made and applied in turn over the same piece of material. The first mask provides the top-level relief and produces flat regions at full depth at any point beyond the dynamic range of the MLT. The next mask etches these newly developed flat regions into the profile at the next level of depth, creating a pattern with a larger peak to valley height. The process is repeated until the full pattern is etched. The key to success here is accurate mask positioning.

\section{CONCLUSION}

MLT manufacturing offers a rapid and inexpensive means to fabricate small custom components for adaptive optics, bypassing many of the normal constraints and costs of manufacturing in small quantities. It is likely that these components will be most valuable as prototypes in the laboratory where experimental work is facilitated by the rapid availability of parts fabricated to order, but we anticipate that there will be instances in which fielded AO systems will also benefit from one-off custom optics that can be made at low cost. The technique is particularly well suited to components that are difficult to make with standard optical fabrication techniques, such as the lenslet arrays, phase screens, and pyramids addressed here.

\section{ACKNOWLEDGMENTS}

We thank Jared Males for the use of his laboratory and supercontinuum laser for the testing of chromatic effects on our diffractive components.

\section{REFERENCES}

[1] Shiono, T., Setsune, K., Yamazaki, O., and Wasa, K., "Rectangular-apertured micro-Fresnel lens arrays fabricated by electron-beam lithography," Appl. Opt. 26, 587-591 (1987) 
[2] Jahns, J. and Walker, S. J., "Two-dimensional array of diffractive microlenses fabricated by thin film deposition," Appl. Opt. 29, 931-936 (1990)

[3] Tyson, R.K. and Frazier, B. W., [Field Guide to Adaptive Optics, Second Edition], SPIE Press, Bellingham, 38 (2012)

[4] Jean, M. A., Bronson, R., Scott, R. P., Hart, M., Johnson, L., and Milster, T., "Multiwavelength Characterization of Multi-Order Diffractive Lenslet Arrays," in Optical Design and Fabrication 2017 (Freeform, IODC, OFT), OSA Technical Digest (online) (Optical Society of America, 2017), paper OW2B.4.

[5] Zhao, Y., Ding, X., Wang, K., Wei, H., Yang, H., Cai, D., "Diffractive pyramid wave-front sensor used for adaptive optics," Proc. SPIE 8420, 842009 (2012)

[6] Diolaiti, E. et al., "Some novel concepts in multipyramid wavefront sensing," Proc. SPIE 4839, 299 (2003) 